\title{
Influence of Energy and Temperature in Cluster Coalescence Induced by Deposition
}

\author{
J. C. Jiménez-Sáez, ${ }^{1}$ A. M. C. Pérez-Martín, ${ }^{2}$ and J. J. Jiménez-Rodríguez ${ }^{2}$ \\ ${ }^{1}$ Departamento de Física y Química Aplicadas a la Técnica Aeronáutica, EIAE, Universidad Politécnica de Madrid (UPM), \\ 28040 Madrid, Spain \\ ${ }^{2}$ Departamento de Física Aplicada III (Electricidad y Electrónica), Facultad de Ciencias Físicas, \\ Universidad Complutense de Madrid (UCM), 28040 Madrid, Spain \\ Correspondence should be addressed to J. C. Jiménez-Sáez, jc.jimenez@upm.es
}

Received 8 November 2012; Accepted 27 November 2012

Academic Editor: Dilip Kanhere

Copyright (๑) 2012 J. C. Jiménez-Sáez et al. This is an open access article distributed under the Creative Commons Attribution License, which permits unrestricted use, distribution, and reproduction in any medium, provided the original work is properly cited.

Coalescence induced by deposition of different $\mathrm{Cu}$ clusters on an epitaxial Co cluster supported on a $\mathrm{Cu}(001)$ substrate is studied by constant-temperature molecular dynamics simulations. The degree of epitaxy of the final system increases with increasing separation between the centres of mass of the projectile and target clusters during the collision. Structure, roughness, and epitaxial order of the supported cluster also influence the degree of epitaxy. The effect of energy and temperature is determinant on the epitaxial condition of the coalesced cluster, especially both factors modify the generation, growth and interaction among grains. A higher temperature favours the epitaxial growth for low impact parameters. A higher energy contributes to the epitaxial coalescence for any initial separation between the projectile and target clusters. The influence of projectile energy is notably greater than the influence of temperature since higher energies allow greater and instantaneous atomic reorganizations, so that the number of arisen grains just after the collision becomes smaller. The appearance of grain boundary dislocations is, therefore, a decisive factor in the epitaxial growth of the coalesced cluster.

\section{Introduction}

Intercluster coalescence is a process extensively studied by using molecular dynamics [1-4] or by macroscopic models of sintering [5]. The underlying physical phenomenon is the capillarity since the surface free energy is reduced. Coalescence of supported clusters is of great importance in the field of surface nanostructuring [6]. In fact, the understanding of coalescence is of primary importance for understanding the structure of cluster-assembled materials, and, therefore, for controlling a number of physical and mechanical properties of thin films. This type of materials can be grown by the low-energy deposition of preformed clusters containing hundreds or thousands of atoms on a surface [7]. Lange and Kellett [8] indicated that a further lowering of the free energy would require the recrystallization of some particles in order to remove some of the grain boundaries between coalesced clusters. In heterocoalescence processes, the origin of these grain boundaries may reside in the crystallographic mismatch. Many groups have performed crystallite rotation techniques in order to investigate the reorientation during sintering for metals [1] and oxide particles [9]. For metals, the mechanism of reorientation should also take into account the formation and migration of grain-boundary dislocations.

In this work, in contrast to other investigations, the heterocoalescence induced by collision between different nanoparticles is discussed by analyzing the grain growth and the structure of the coalesced cluster at different temperatures and projectile-cluster energies. Specifically, $\mathrm{Cu}$ and $\mathrm{Co}$ clusters, the latter supported on a $\mathrm{Cu}(001)$ substrate, have been used. Cobalt clusters embedded or supported in a copper matrix constitute an attractive system, because it displays important magnetic properties [10]. Initially, a randomly oriented Co cluster (target) was deposited on a $\mathrm{Cu}(001)$ substrate. Next, a randomly oriented $\mathrm{Cu}$ cluster (projectile) at different energies was deposited on the former 
colliding with this. Evidently, in the actual coalescence, substrate plays the important role of ensuring thermalization. The centre-of-mass separation between the projectile and target clusters at the instant of collision was varied. The degree of epitaxy of both clusters upon impact as a function of this separation has been investigated. A statistical study has been necessary to elucidate differences in this magnitude. Changes with temperature and deposition energy due to the activation of grain-boundary movement and grain inner rotation have also been analyzed.

\section{Model and Analysis Methods}

Equations of motion were derived from the Lagrangian proposed by Parrinello and Rahman [11]. The simulation time was about 100 ps, although some cases were run until 1 ns to check the stability of results. The potential was formulated in the second-moment tight-binding approximation (TBSMA) by Levanov et al. [12] to fit experimentally cohesive energies and elastic constants for bulk $\mathrm{Cu}$ (faced-centred cubic, fcc phase) and Co (hexagonal close-packed, hcp), as well as, $a b$ initio interaction energies for small Co clusters on Cu surfaces.

The number of $\mathrm{Cu}$ substrate unit cells, $30 \times 30 \times 10$, was larger than that used in another similar simulation works [13]. Besides, simulation times obtained with this size allowed us to make an incipient statistical analysis of epitaxy. Periodic boundary conditions were used in the $x$ - and $y$-directions parallel to the free (001) surface, and nonperiodic boundary conditions, in the normal direction ( $z$-direction). The three bottom layers of this direction were fixed, and the five ones above these were thermal control layers, which were assumed to be an ideal heat sink and maintained a constant substrate temperature. The algorithm of temperature control was the Nosé-Hoover thermostat [14]. The crystalline structure of the projectile and target clusters before their deposition was one of the most stable [15], hexagonal faceted cuboctahedron (CO), also so-called Wulff polyhedron, of 586 atoms and fcc phase [16].

First of all, a Co cluster (target) was deposited epitaxially by choosing suitable conditions of impinging energy [17]. Next, a Cu cluster (projectile) was deposited colliding with the previous supported cluster. Temperature and deposition energy were varied in order to analyze its influence on the degree of epitaxy of the resulting system. In order to reproduce experimental conditions, the projectile cluster was rotated randomly. In addition, statistical averages were accomplished for about ten cases (different cluster rotations or Euler angles). The number of simulated cases does not allow to obtain exact values of the final degree of epitaxy (with low dispersion), since that was not our aim, but rather to get guiding values. The initial position of the projectile cluster is determined by varying the $x$-coordinate of its centre of mass relative to the same coordinate of the supported cluster. The difference between both coordinates is the impact parameter, which has been normalized by the sum of the radii of the two colliding clusters, parameter $d_{r}$. Hence, a relative distance of more than 1 corresponds to a situation where the two clusters are not touching at the moment of impact.

Epitaxial matching between a nanocluster and the substrate has been quantified by a previously defined epitaxy factor, $f_{\text {epi }}[18]$. In a supported cluster, $f_{\text {epi }}$ measures the average angular displacement of first-neighbour position vectors (relative to each atom), as compared to what these vectors would be if this atom would be in a perfect continuation of the substrate lattice. In a previous work, it was shown that a cluster with a value of $f_{\text {epi }}<1$ could be considered epitaxial [17]. A program to monitor the grain growth was developed. This program uses, first of all, the common neighbour analysis (CNA) [19] to find out the crystalline structure around an atom: fcc or hcp. Next, the lattice unit vectors for every atom are determined; taking into account that when there are several options, the program selects that set of directions found more frequently in neighbouring atoms. At any given time, a map of grains in the cluster is generated by grouping together similar sets of lattice unit vectors. Finally, the program connects the grains at that instant with the existing ones at a previous moment. If a grain can be associated with several of them due to its nearness (separation of centres of mass), the one with the same crystallographic orientation is chosen firstly. Unless otherwise stated, simulations will be accomplished at $300 \mathrm{~K}$, with a deposition energy of $0.25 \mathrm{eV} /$ atom $(871 \mathrm{~m} / \mathrm{s})$ on a target cluster with an initial $f_{\text {epi-factor }}=0.43$ (reference case).

\section{Results and Discussion}

First of all, the influence of initial epitaxial order and surface structure of the target Co cluster on the alignment process was analysed. A projectile $\mathrm{Cu}$ cluster was deposited on three different systems. In the case of the supported target cluster,

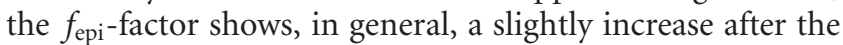
collision; however, this cluster does not lose its epitaxial condition. The key of the epitaxial growth, therefore, resides in the projectile cluster. The $f_{\text {epi }}$-factor for this cluster as a function of the impact parameter after the collision is shown in Figure 1(a). In spite of the large standard deviation, if the target cluster does not show an initial epitaxial structure (case C, initial $f_{\text {epi }}=1.11$ ), the average values of $f_{\text {epi }}$ increase in relation to the reference Case B, specifically, for low impact parameters. In any case, epitaxial growth $\left(f_{\text {epi }}<1\right)$ is not observed for parameters in this order in both cases (B and $\mathrm{C})$. The main reason is that the $\mathrm{Cu}$ substrate does not exert a significant alignment influence on the projectile $\mathrm{Cu}$ cluster [20] since this does not touch the surface; apart from the existing lattice misfit between both clusters (2\%). The average potential energy of the coalesced cluster, after an initial fast increase, decreases as both clusters merge. And, in fact, if the projectile cluster binds with the substrate, that is, if the impact parameter is large enough, this energy reaches a lower limit: the system reaches a completely epitaxial configuration. In general, the final potential energy of the system is higher if grains are formed in the cluster [21]. 


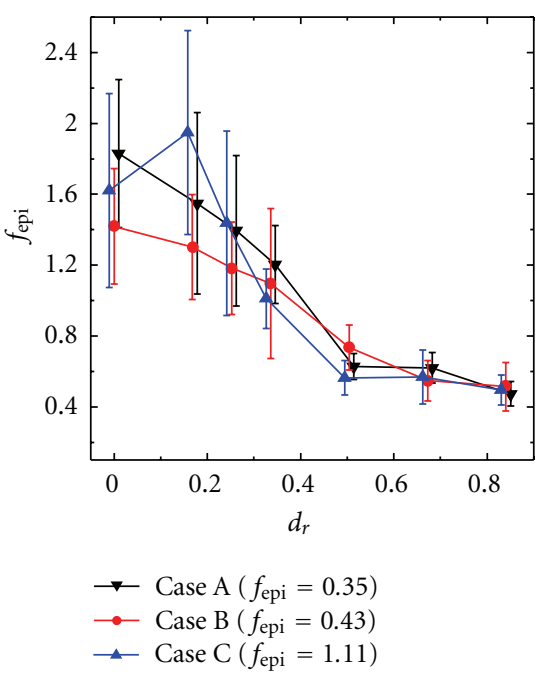

(a)

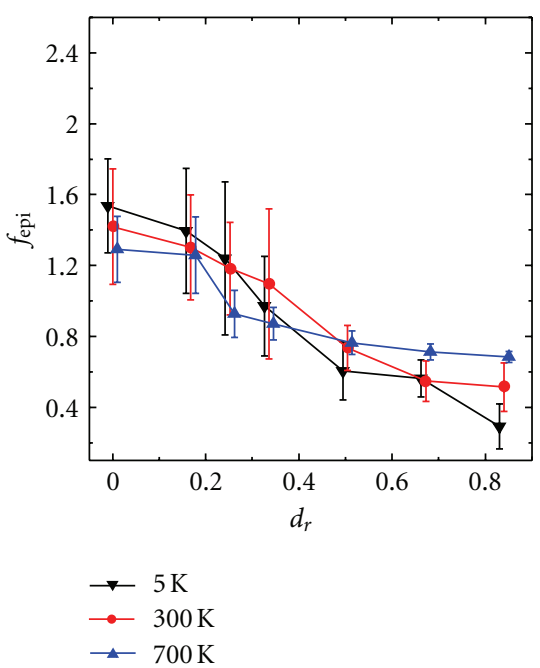

(b)

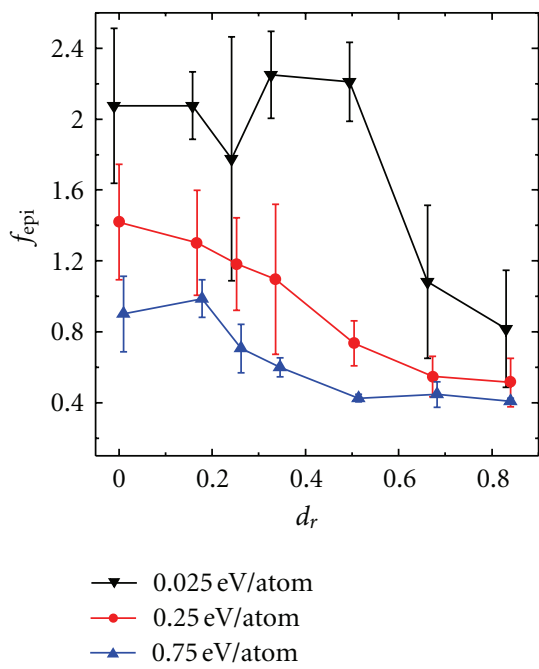

(c)

FIGURE 1: $f_{\text {epi }}$-factor after the collision for the projectile cluster as a function of the impact parameter, $d_{r}$, for three cases with different target cluster (a), at three different temperatures (b), and for three different deposition energies (c).

In order to analyse the influence of initial surface morphology of the target cluster, a Mackay icosahedral cluster of 561 atoms with fcc phase was deposited as target cluster. After its deposition, the structure of this cluster was extremely epitaxial (case A, initial $f_{\text {epi }}=0.35$ ). However, the number of surface atoms corresponding to $\{111\}$ and $\{100\}$ surfaces was large, $28 \%$ and $16 \%$ respectively, against $23 \%$ and $5 \%$ for the reference case. Therefore, the initial surface of this cluster was formed to a greater extent by stable plane surfaces, unlike the reference target cluster, that was mainly composed of rounded and rough surfaces lacking in structure. These plane-surface structures make the recovery of the epitaxial order difficult. Thus, in Figure 1(a),

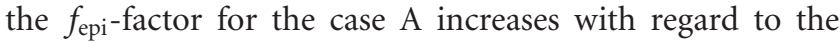
reference case, especially, for low impact parameters. Usually, interaction energy increases near steps (and other roughness) due to the higher atomic coordination available at the step edge [22]. This higher energy causes a noticeable increase in the initial deformation of the cluster upon impact, which directly results in structures with a higher degree of final ordering [22].

Temperature is another factor influencing the epitaxial growth [17]. In Figure 1(b), the degree of epitaxy reached by the projectile cluster is shown at different temperatures. The difference between average values at $5 \mathrm{~K}$ and $700 \mathrm{~K}$ is not excessively large. However, it is worth emphasizing the change of tendency of these curves for intermediate impact parameters. Thus, for low impact parameters, if temperature increases, the effect of thermal activation of grain-boundary movement and grain reorientation collaborates to a greater epitaxial order, decreasing the value of $f_{\text {epi }}[21]$; on the contrary, for high impact parameters, the greater thermal vibration in epitaxial conditions increases the disorder and, therefore, decreases the degree of epitaxy. The average potential energy of the coalesced cluster also shows differences. During the impact, for low impact parameters, the increase relative to the initial value of this energy is the same at $5 \mathrm{~K}$ and $700 \mathrm{~K}$ (collision change). However, the relative decrease at subsequent time instants is larger for high temperatures (due to the thermal activation and, therefore, to the better final alignment with the substrate). Nevertheless, for growing impact parameters, the difference for high and low temperatures is increasingly smaller.

Crystallinity of the coalesced cluster also depends on the deposition energy. In Figure $1(\mathrm{c})$, the $f_{\text {epi-factor is shown }}$ at different energies. As the deposition energy increases, alignment with the substrate is greater, eventually resulting in completely epitaxial films at sufficiently high energies [21]. Deposition at $0.75 \mathrm{eV} /$ atom $(1509 \mathrm{~m} / \mathrm{s})$ gives rise to epitaxial clusters at any impact parameter; even the statistical deviation for different rotations is very low. Just the opposite happens in the $0.025-\mathrm{eV} /$ atom case $(276 \mathrm{~m} / \mathrm{s})$. At low energy, the projectile cluster only reaches the surface for high impact parameters, about 0.5; since, due its size, the target cluster easily slows down its advance. Unlike, at $0.75 \mathrm{eV} /$ atom, the projectile cluster comes into contact with the surface for impact parameters around 0.2. These facts clearly determine the shape of $f_{\text {epi-curves: }}$ at low energy, their fall, at high impact parameters, is steep; at high energy, they begin to decrease smoothly at quite lower impact parameters.

Before the coalescence process between clusters takes place, a much faster reorientation process of adjacent nanoparticles is necessary [9]. In fact, if reorientation is suppressed, the lattice mismatch opposes the complete coalescence, in agreement with the predictions of Lange and Kellett [8]; even in many cases a complete coalescence was inhibited by a grain boundary so that the system remained in a dumbbell-like shape (in coalescence processes without deposition) [9]. In Figure 2, the time evolution of the number of atoms (in percentage) in different grains of the 


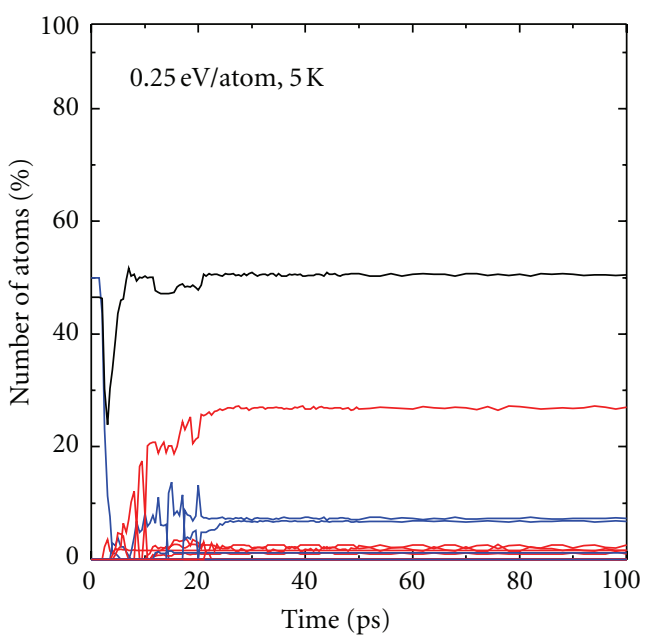

(a)

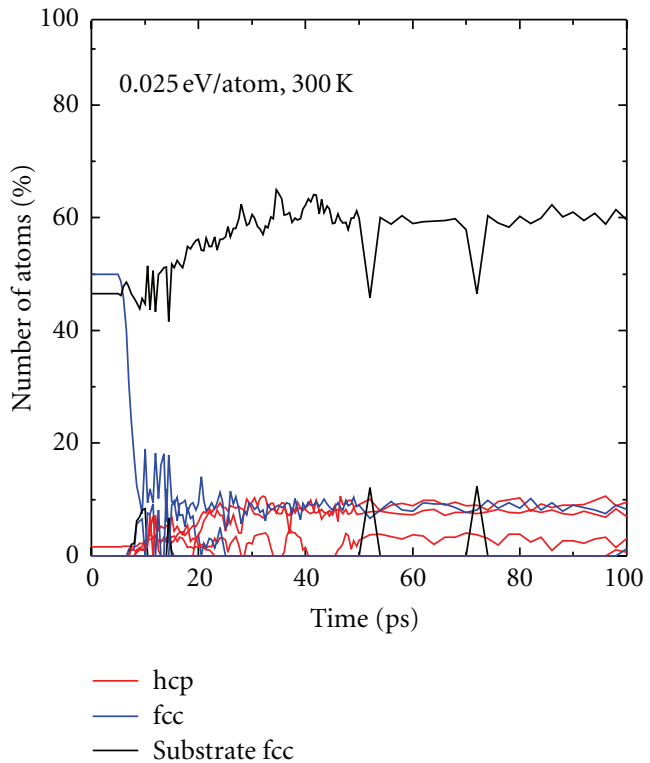

(c)

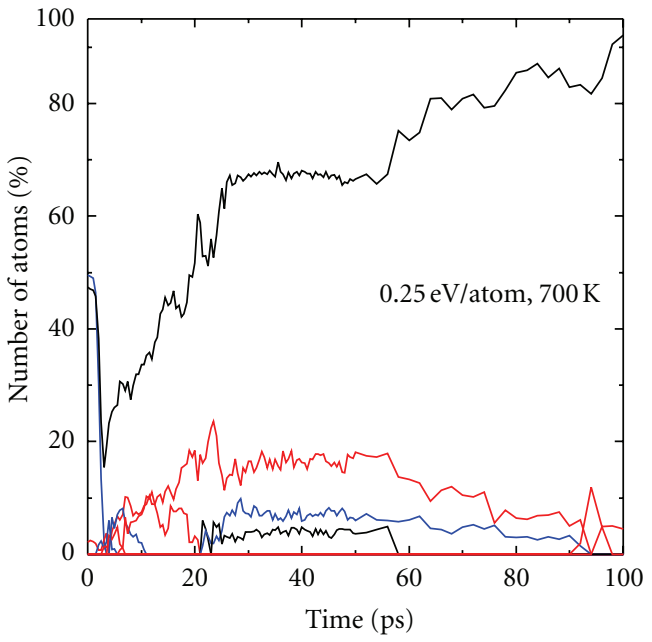

(b)

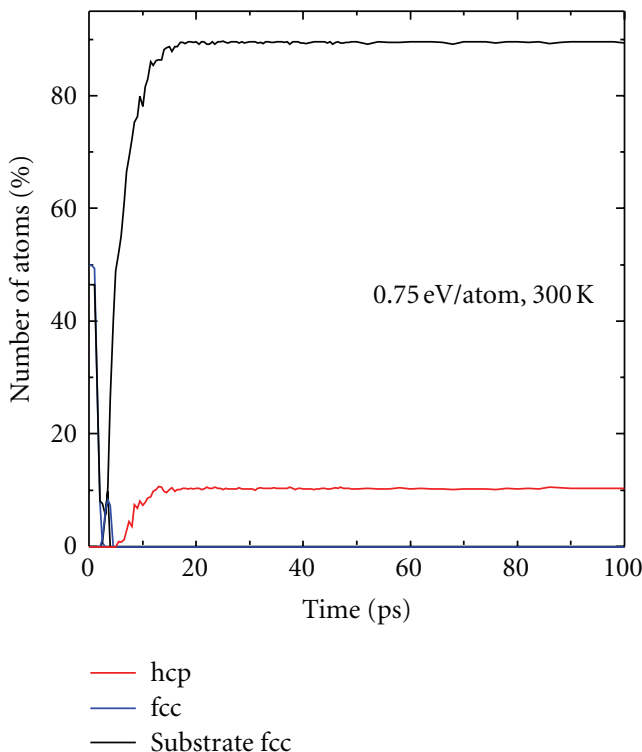

(d)

FIGURE 2: Number of atoms as a function of the simulation time in each grain at different temperatures ( 5 and $700 \mathrm{~K})$ and energies ( 0.025 and $0.75 \mathrm{eV} /$ atom) for the case of $d_{r}=0.26$. The main grain (substrate fcc), hcp grains, and other fcc grains are distinguished.

system formed by both clusters is shown for the case of $d_{r}=0.26$. At low temperature $(5 \mathrm{~K})$, there are hardly changes in the number of atoms of each grain, or in the number of grains, which is quite large. Atoms in the main grain fundamentally come from the epitaxial target cluster. The 3D image is more conclusive: three hcp nanograins, that is, stacking faults on $\{111\}$ planes slow down the advance of the fcc main grain, oriented just like the substrate (see Figure 3) and with the lowest average potential energy (higher binding energy). This binding energy for every grain practically stays constant, except little fluctuations. 1-layer hcp grain corresponds to a stacking sequence $\mathrm{ABCBCA}$, and 2-layer hcp grain corresponds to a sequence $\mathrm{ABCBCBA}$. The 700-K case shows a different behavior: the number of atoms in the main grain grows continuously, whereas the rest of nanograins loses atoms or disappears. This atomic incorporation induced by thermal activation (or change in the stacking) means to decrease the binding energy in the main grain, since $\mathrm{Cu}$ atoms of the projectile cluster are added (cohesive energy is higher for Co than for $\mathrm{Cu}$ ). Twinning dislocations moves through the cluster changing the stacking in such a way that nonepitaxial layers becomes epitaxial [23].

In the case of deposition at low energy (and $300 \mathrm{~K}$ ), evolution of the inner structure of the coalesced cluster is similar to the low-temperature case (stacking faults slowing down the advance of the main grain, see Figure 3), with the difference that this grain slightly grows due to the light thermal activation, which produces a little change in the energy levels of the involved grains. At high energy, the growth of the main grain is almost instantaneous after the 

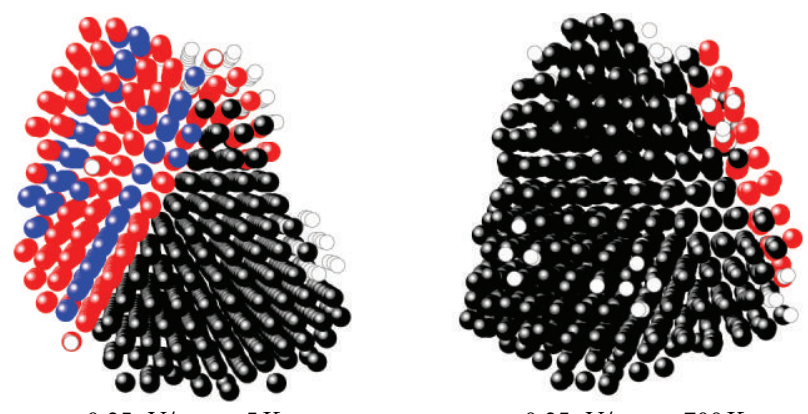

$0.25 \mathrm{eV} /$ atom, $5 \mathrm{~K}$

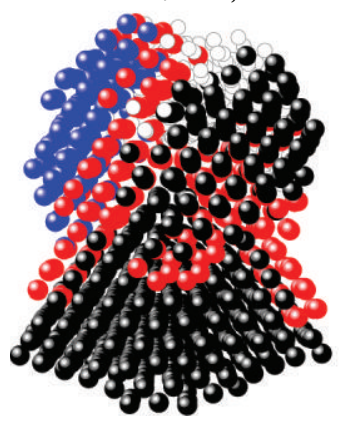

$0.025 \mathrm{eV} /$ atom, $300 \mathrm{~K}$

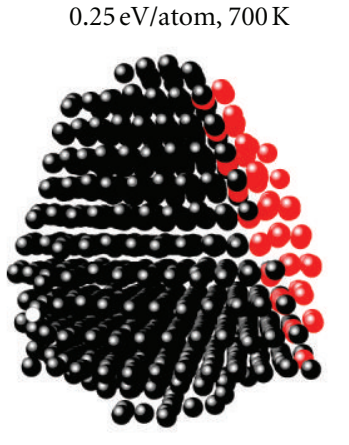

$0.75 \mathrm{eV} /$ atom, $300 \mathrm{~K}$

Figure 3: Snapshot of the final state at different temperatures and energies for an impact parameter of $d_{r}=0.26$ distinguishing the main grain (black), hcp grains (red), other fcc grains (blue), and the rest of atoms (without colour). The viewing direction is close to the $\langle 110\rangle$.

collision. Only, a surface hcp nanograin stays stable at the end of the process (see Figure 3), as in the case of high temperature. Besides, both surface grains have the same unit lattice vectors. An analysis of the average potential energy as a function of the simulation time reveals that the disappearance of grains is consequence, in part, of the maximum in this energy upon impact (collision stress), since at low energy this peak does not exist. In fact, this peak must be high enough to allow the potential energy to reach a low enough value (epitaxial ordering). The other cause of epitaxial matching is the mixing. At $0.75 \mathrm{eV} /$ atom, the layer of the coalesced cluster next to the substrate (interface layer) is populated by about $22 \%$ of $\mathrm{Cu}$ atoms from the projectile cluster; however, at $0.25 \mathrm{eV} /$ atom, this number is reduced at about $4 \%$; and at $0.025 \mathrm{eV} /$ atom at $0 \%$. Thus, the stress by lattice misfit decreases notably at high energy.

It is interesting to discuss more in detail the time evolution of the $700 \mathrm{~K}$ case (see Figure 4). Immediately after the collision ( $\sim 10 \mathrm{ps})$, two 2-layer hcp nanograins are developed. One of them is placed at the interface between clusters, inside $\mathrm{Co}$ and $\mathrm{Cu}$, parallel to a $(1,-1,1)$ plane (not shown in figure); and the other is placed next to the interface, inside the Co cluster, parallel to a $(1,1,1)$ plane. The collision stress, the lattice misfit, and the trend toward the hcp phase of Co crystallites (the used cut-off radius favours this phase) [24] would be the cause of the appearance of these nanostructures. After $20 \mathrm{ps}$, the latter nanograin has grown and includes 4 layers at both sides of the interface. Beside this, in the $\mathrm{Cu}$ cluster and reaching its surface, a substrateoriented fcc grain begins to be developed. On the other

hand, the former hcp nanograin, more irregular than the latter, is being absorbed mainly by the main grain. At 40 ps, this nanograin has disappeared and its atoms have been incorporated by the neighbouring grains in a clear process of grain-boundary migration [25]. For its part, the latter hcp nanograin loses one (111) layer, specifically, that in contact with the main grain that is absorbed by this. Besides, an intermediate layer of this grain at the $\mathrm{Cu}$ interface moves to generate a stacking fault, CBCAC, more stable in Cu than an hcp nanograin, although its structure (BCA) is not substrate oriented. Beside this, the surface substrate-oriented grain has grown slightly. At $60 \mathrm{ps}$, the substrate-oriented grain and the main grain have been linked to each other underneath, given that the main grain has completely incorporated two horizontal files in contact with the substrate. This atomic relocation continues upwards, although not in a vertical way, but in a $(1,1,-2)$ direction. Thus, the main grain continues adding atoms. At $90 \mathrm{ps}$, a wedge-shaped structure can be distinguished at the top of the Cu cluster. Its surface is formed by 2-layer hcp nanograins placed in different $\{111\}$ planes. In turn, inside, there is other wedge-shaped structure, but this is fcc. Therefore, part of the hcp nanograin begins to grow, but in layers parallel to a $(-1-11)$ plane (instead of a (111) plane); and in the interior, the fcc nanograin begins to rotate toward a substrate-oriented structure. At $96 \mathrm{ps}$, this fcc grain is linked to the main grain. As well as, the hcp grain surrounded by the main grain begins to be absorbed by this. Finally, only stays the grain parallel to the $(-1-11)$ plane, which rotates and recovers its initial crystallographic orientation, and moves by the surface in parallel layers to (111) planes.

\section{Conclusions}

The effect of temperature, deposition energy, or morphology of the target cluster is analysed in the crystallographic reorientation process induced by deposition of different $\mathrm{Cu}$ clusters on an epitaxial Co cluster supported on a $\mathrm{Cu}(001)$ substrate. Degree of epitaxy of the projectile cluster increases with increasing impact parameter between both clusters. However, changes in this dependence are observed by modifying some variables. Below, we summarize the main results.

(i) Degree of epitaxy for low impact parameters increases if the target cluster improves its epitaxial order or increases its surface roughness.

(ii) Epitaxial order also increases for low impact parameters with increasing temperature since thermal activation contributes to the grain-boundary migration and the grain reorientation. For high impact parameters, thermal vibration decreases the epitaxial ordering.

(iii) Deposition energy is the factor with the most weight, since, at high energies, coalesced cluster reaches an epitaxial configuration even for low impact parameters. Collision stress and mixing are the responsible for the epitaxial matching. 


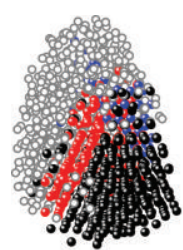

$10 \mathrm{ps}$

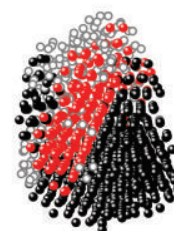

$20 \mathrm{ps}$

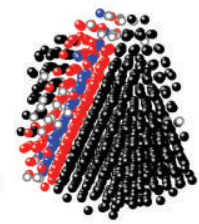

$40 \mathrm{ps}$

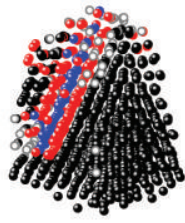

$60 \mathrm{ps}$

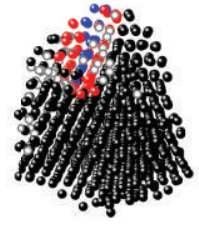

$80 \mathrm{ps}$

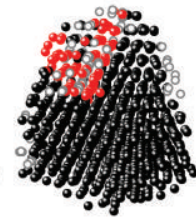

90 ps

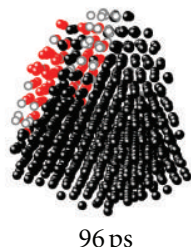

$96 \mathrm{ps}$

FIGURE 4: Snapshots of the time evolution for the $700-\mathrm{K}$ case $(0.25 \mathrm{eV} /$ atom) with a low-intermediate impact parameter (0.26). The main grain (black), hcp grains (red), other fcc grains (blue), and rest of atoms (without colour) are distinguished. The viewing direction is close to the $\langle 110\rangle$.

A time analysis of grain boundaries shows, at low temperature or energy, that there is hardly changes in the number of atoms of each grain, or in the number of grains, which is, in addition, quite large. Stacking faults on $\{111\}$ planes slows down the advance of the substrate-oriented main grain. At high temperature, twinning dislocations moves through the cluster changing the stacking, so that nonepitaxial layers become epitaxial; and high energy, the initial high stress minimizes the appearance of these defects. After the collision, hcp nanograins grow mainly at the interface. Sometimes, these nanograins can disappear by grain-boundary migration, absorbed by other grains, and others; they become stacking faults (more stable in $\mathrm{Cu}$ ). These faults move to the surface, being able to rotate their orientation or are absorbed by the main grain, changing their structure.

\section{Acknowledgments}

This work is a part of the Research Group Program, Bioelectromagnetism Group 910305 of UCM, which is financially supported by UCM-Santander.

\section{References}

[1] S. Arcidiacono, N. R. Bieri, D. Poulikakos, and C. P. Grigoropoulos, "On the coalescence of gold nanoparticles," International Journal of Multiphase Flow, vol. 30, no. 7-8, pp. 979994, 2004.

[2] E. Blaisten-Barojas and M. R. Zachariah, "Molecular-dynamics study of cluster growth by cluster-cluster collisions," Physical Review B, vol. 45, no. 8, pp. 4403-4408, 1992.

[3] M. M. Mariscal, S. A. Dassie, and E. P. M. Leiva, "Collision as a way of forming bimetallic nanoclusters of various structures and chemical compositions," Journal of Chemical Physics, vol. 123, no. 18, Article ID 184505, pp. 1-6, 2005.

[4] G. Li, Q. Wang, T. Liu, K. Wang, and J. He, "Molecular dynamics simulation of the melting and coalescence in the mixed Cu-Ni nanoclusters," Journal of Cluster Science, vol. 21, no. 1, pp. $45-55,2010$.

[5] F. A. Nichols and W. W. Mullins, "Morphological changes of a surface of revolution due to capillarity-induced surface diffusion," Journal of Applied Physics, vol. 36, no. 6, pp. 18261835, 1965.

[6] P. Jensen, "Growth of nanostructures by cluster deposition: experiments and simple models," Reviews of Modern Physics, vol. 71, no. 5, pp. 1695-1735, 1999.
[7] A. Perez, P. Melinon, V. Dupuis et al., "Cluster assembled materials: a novel class of nanostructured solids with original structures and properties," Journal of Physics D, vol. 30, no. 5, pp. 709-721, 1997.

[8] F. F. Lange and B. J. Kellett, "Thermodynamics of densification: II. Grain growth in porous compacts and relation to densification," Journal of the American Ceramic Society, vol. 72, no. 5, pp. 735-741, 1989.

[9] R. Theissmann, M. Fendrich, R. Zinetullin, G. Guenther, G. Schierning, and D. E. Wolf, "Crystallographic reorientation and nanoparticle coalescence," Physical Review B, vol. 78, no. 20, Article ID 205413, 10 pages, 2008.

[10] J. C. Cezar, H. C. N. Tolentino, and M. Knobel, "Structural, magnetic, and transport properties of Co nanoparticles within a Cu matrix," Physical Review B, vol. 68, no. 5, Article ID 054404, 10 pages, 2003.

[11] M. Parrinello and A. Rahman, "Polymorphic transitions in single crystals: a new molecular dynamics method," Journal of Applied Physics, vol. 52, no. 12, pp. 7182-7190, 1981.

[12] N. A. Levanov, V. S. Stepanyuk, W. Hergert et al., "Energetics of Co adatoms on the $\mathrm{Cu}(001)$ surface," Physical Review B, vol. 61, no. 3, pp. 2230-2234, 2000.

[13] C. K. Chen and S. C. Chang, "Modelling transport phenomena and epitaxial behaviours of cluster-surface collisions via molecular dynamics simulations," Nanotechnology, vol. 17, no. 20, pp. 5051-5062, 2006.

[14] W. G. Hoover, "Canonical dynamics: equilibrium phase-space distributions," Physical Review A, vol. 31, no. 3, pp. 1695-1697, 1985.

[15] O. Kitakami, H. Sato, Y. Shimada, F. Sato, and M. Tanaka, "Size effect on the crystal phase of cobalt fine particles," Physical Review B, vol. 56, no. 21, pp. 13849-13854, 1997.

[16] S. C. Hendy and B. D. Hall, "Molecular dynamics simulations of lead clusters," Physical Review B, vol. 64, Article ID 085425 , 11 pages, 2001.

[17] C. Jiménez-Sáez, A. M. C. Pérez-Martín, and J. J. JiménezRodríguez, "Dependence on temperature and energy of the heteroepitaxy of small metallic nanoclusters," Journal of Nanoscience and Nanotechnology, vol. 11, pp. 8712-8717, 2011.

[18] J. C. Jiménez-Sáez, A. M. C. Pérez-Martín, and J. J. JiménezRodríguez, "Epitaxy of softly deposited small Co nanoclusters on Cu(001) surfaces," Physica Status Solidi A, vol. 205, pp. 1330-1336, 2008.

[19] C. L. Cleveland, W. D. Luedtke, and U. Landman, "Melting of gold clusters," Physical Review B, vol. 60, no. 7, pp. 5065-5077, 1999.

[20] J. C. Jiménez-Sáez, A. M. C. Pérez-Martín, and J. J. JiménezRodríguez, "Nanoparticle heterocoalescence induced by deposition," Physica Status Solidi C, vol. 7, pp. 2600-2603, 2010. 
[21] K. Meinander, J. Frantz, K. Nordlund, and J. Keinonen, "Upper size limit of complete contact epitaxy," Thin Solid Films, vol. 425, no. 1-2, pp. 297-303, 2003.

[22] F. J. Palacios, M. P. Iñiguez, M. J. López, and J. A. Alonso, "Molecular-dynamics study of the structural rearrangements of $\mathrm{Cu}$ and Au clusters softly deposited on a $\mathrm{Cu}(001)$ surface," Physical Review B, vol. 60, pp. 2908-2915, 1999.

[23] T. T. Järvi, A. Kuronen, K. Meinander, K. Nordlund, and K. Albe, "Contact epitaxy by deposition of $\mathrm{Cu}, \mathrm{Ag}, \mathrm{Au}, \mathrm{Pt}$, and $\mathrm{Ni}$ nanoclusters on (100) surfaces: size limits and mechanisms," Physical Review B, vol. 75, no. 11, Article ID 115422, 11 pages, 2007.

[24] F. Cleri and V. Rosato, "Tight-binding potentials for transition metals and alloys," Physical Review B, vol. 48, no. 1, pp. 22-33, 1993.

[25] A. J. Haslam, D. Moldovan, S. R. Phillpot, D. Wolf, and H. Gleiter, "Combined atomistic and mesoscale simulation of grain growth in nanocrystalline thin films," Computational Materials Science, vol. 23, no. 1-4, pp. 15-32, 2002. 

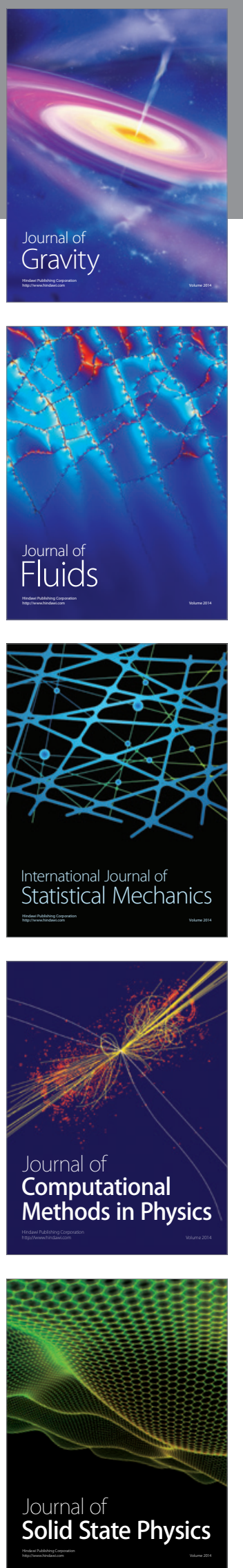

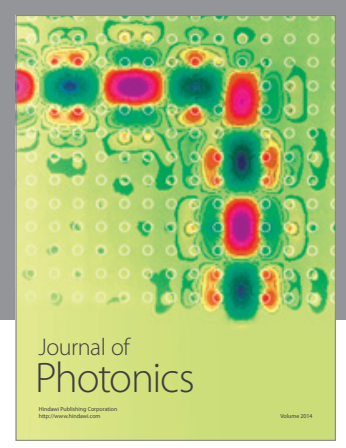

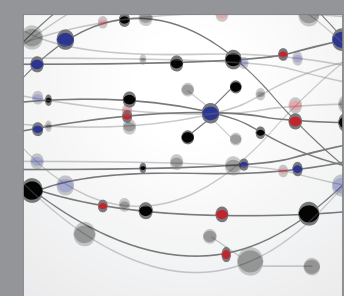

The Scientific World Journal
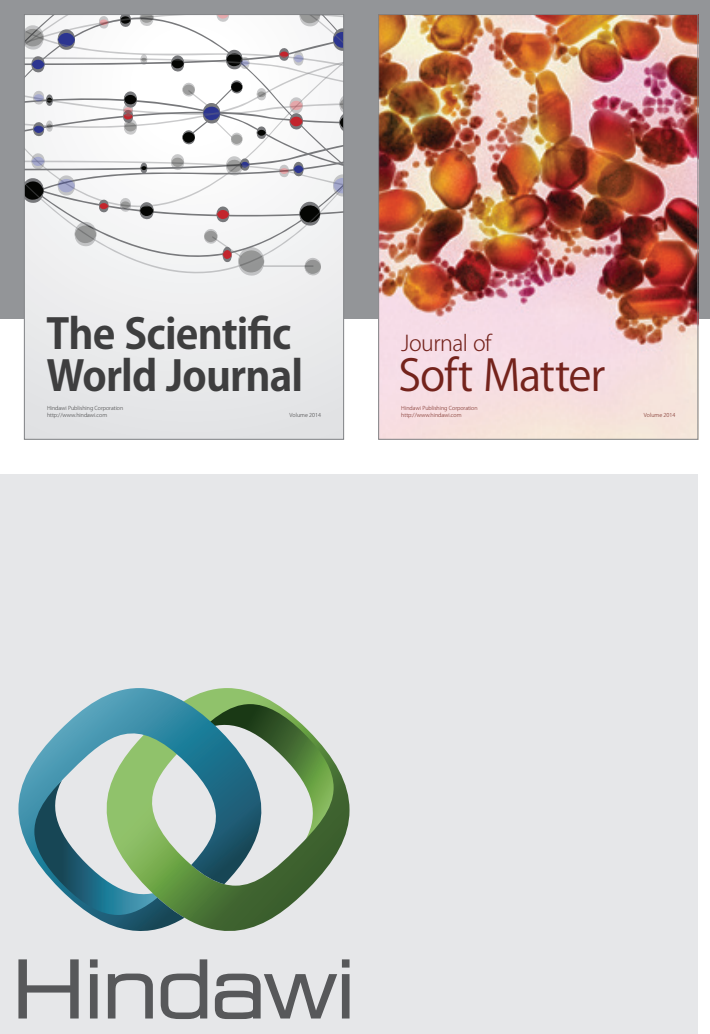

Submit your manuscripts at

http://www.hindawi.com
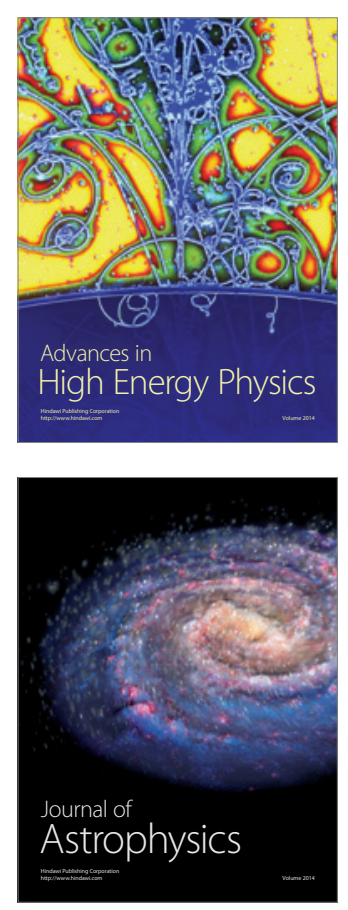
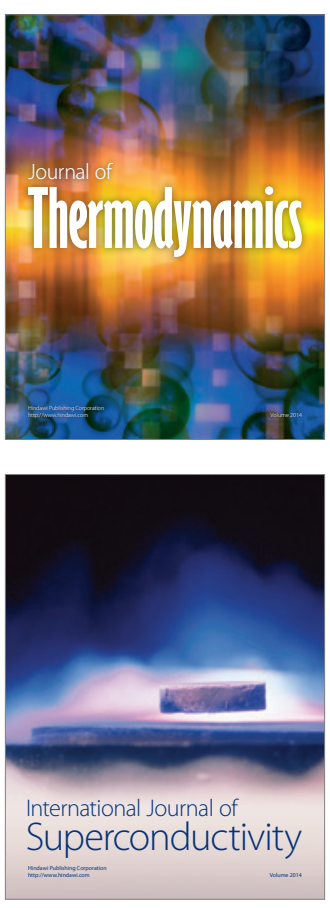
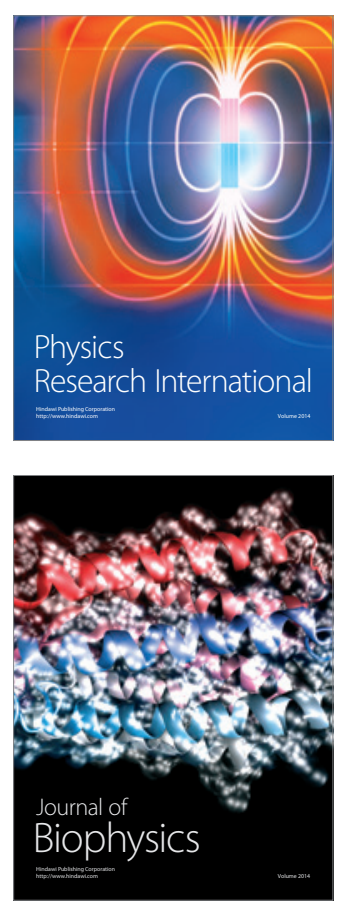
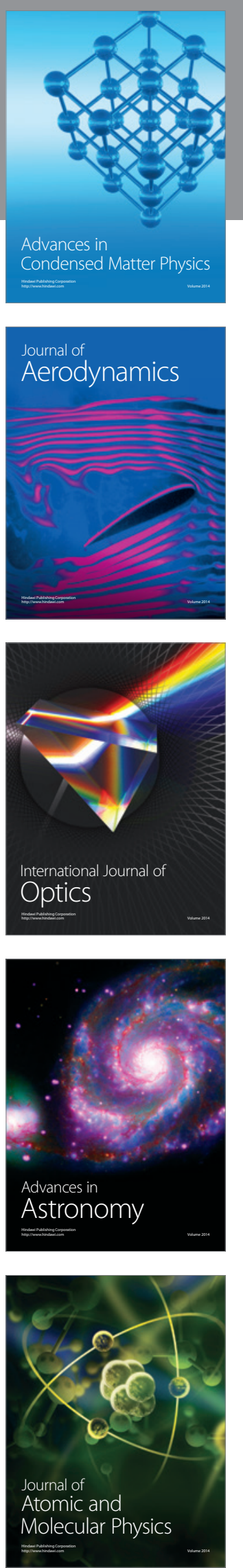